\title{
THE PRESSURE WAVE FORM IN THE PULMONARY ARTERY
}

\author{
BY \\ DAVID B. SHAW \\ From the Cardiac Department, United Bristol Hospitals*
}

Received August 13, 1962

The animal experiments of Howell and Donaldson (1884) and Frank (1895) demonstrated that changes in the conditions of ventricular discharge altered the character of ventricular contraction. More recently Bouchard and Cornu (1954), Rodbard and Shaffer (1956), and Rashkind (1960) have reported modifications of the normal right ventricular pressure wave form in human subjects suffering from diseases affecting the right ventricular outflow tract or pulmonary circulation. It might be expected that the pressure pulse in the pulmonary artery would also be modified by changes in the hæmodynamics of the pulmonary system.

Unfortunately the usual method of recording pulmonary arterial pressure, using a fluid-filled catheter connected to a transducer outside the body, does not give sufficiently accurate and artefactfree tracings to allow the pressure wave form to be studied. To overcome this, Ellis, Gauer, and Wood (1951), and Soulié et al. (1954), developed manometers that were sufficiently small to fit on the end of a cardiac catheter so that they could be introduced directly into the heart chambers. The instrument described by Soulié et al. (1954), which they termed a "micromanometer", consists

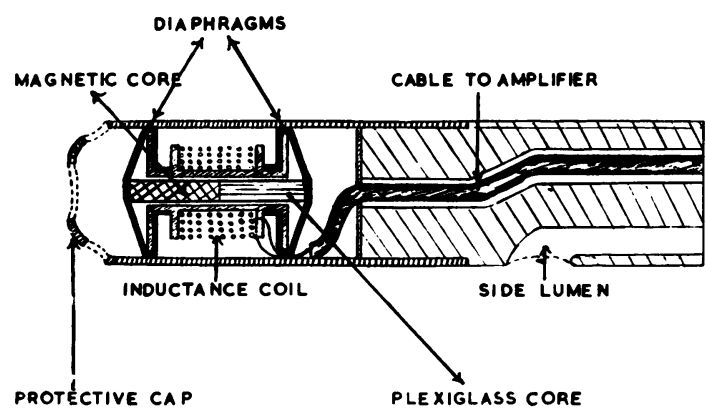

Fig. 1.-The Allard-Laurens micromanometer mounted at the tip of a double lumen catheter (manufactured by Telco of Paris).

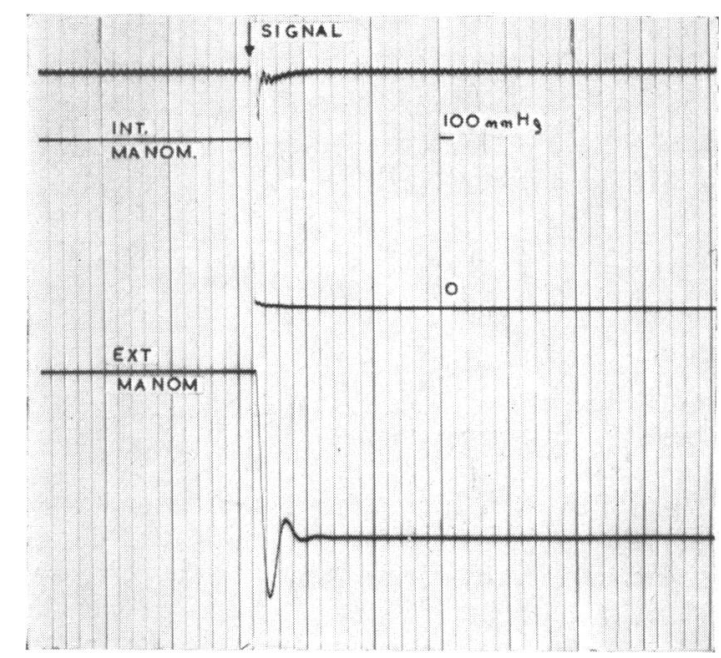

FIG. 2.-A comparison of the response time of the intracardiac micromanometer (int. manom.) with that of a standard N.E.P. manometer at the end of a fluidfilled cardiac catheter (ext. manom.). Both manometers were subjected to a pressure of $100 \mathrm{~mm}$. $\mathrm{Hg}$ suddenly falling to zero (method based on that described by Norman, 1958).

\footnotetext{
* Present address: Cardiopulmonary Unit, University of Colorado Medical Center, Denver, Colorado.
} 
of a transducer that responds to both pressure and sound waves by altering its electrical inductance (Fig. 1). These alterations are conducted to an amplifier outside the body by a cable running in one lumen of a double lumen size 8 cardiac catheter, the second lumen being left patent so that intracardiac pressures can be recorded with an external manometer in the usual way. The amplifier splits the signal into two bands so that pressure and sound are recorded separately. Fig. 2 compares the rapid response and lack of inertia or artefact in the recording from the micromanometer with the delayed response and series of damped pressure oscillations obtained with the usual type of external transducer at the end of a fluid-filled catheter.

The work presented here consists of a study of the pulmonary artery pressure wave form in 43 subjects using an Allard-Laurens micromanometer. To decrease the effect that variations in the power and character of the cardiac contraction might have upon the wave form, only the diastolic portion of the pulse has been considered.

\section{SUBJECTS AND METHODS}

The ages of the patients ranged from 5-55 years and all but three were suffering from congenital heart disease (see Tables I and II). For descriptive purposes they have been classified into three groups upon a hæmodynamic basis. Group I contains 23 subjects with an increased pulmonary blood flow (pulmonary: systemic flow ratio of at least 2:1) and normal pulmonary arterial pressure (systolic $30 \mathrm{~mm}$. $\mathrm{Hg}$ or less). Of

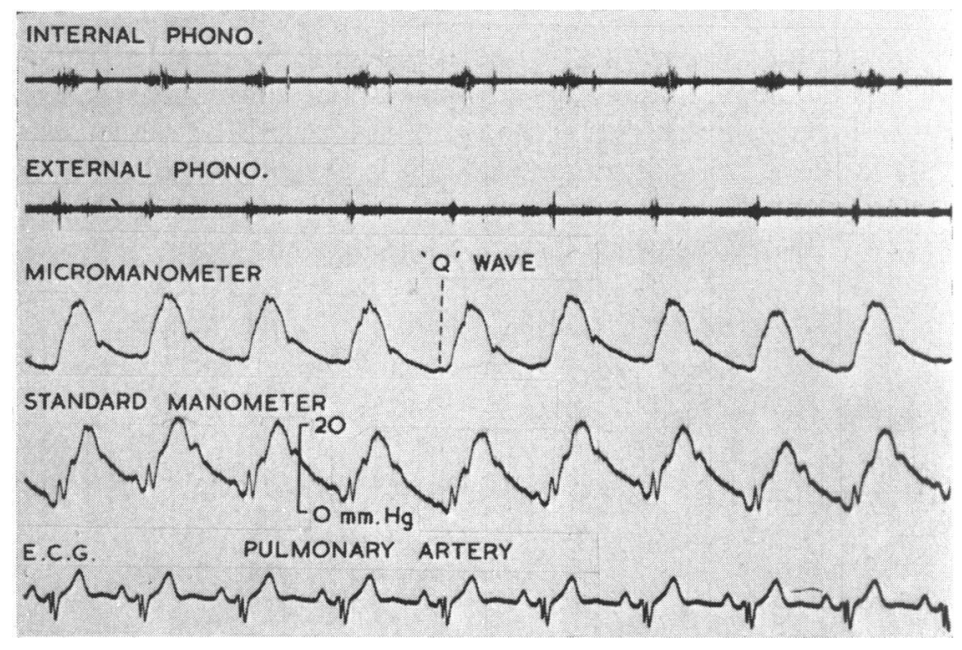

FIG. 3.-Pressure recording obtained from the main pulmonary artery of a patient with an atrial septal defect (Case 20) showing a small presystolic wave (labelled $\mathbf{Q}$ wave). At the slow paper speed of this recording accurate timing of the presystolic wave is impossible, but using a faster speed (8 $\mathrm{cm}$. per second) the peak of the presystolic wave was seen to be 0.16 second after the start of the $P$ wave of the electrocardiogram.

these 16 had normal pulmonary outflow tracts (Group IA), and 7 had pulmonary valvular or infundibular stenosis (Group IB). Group II contains $\mathbf{8}$ subjects with a pulmonary arterial systolic blood pressure of 40 $\mathrm{mm}$. Hg or more (pulmonary hypertension, group IIA) and 8 with pulmonary stenosis and normal pulmonary arterial pressure and blood flow (Group IIB). In the patients with pulmonary stenosis the pressure difference between the two sides of the pulmonary valve was less than $50 \mathrm{~mm}$. $\mathrm{Hg}$ in three cases (mild stenosis), $50-100 \mathrm{~mm}$. $\mathrm{Hg}$ in three cases including one with Fallot's tetralogy (moderate stenosis), and greater than $100 \mathrm{~mm}$. $\mathrm{Hg}$ in two cases (severe stenosis). Group III contains four subjects with various lesions that do not come within the criteria for Groups I and II.

Recordings of the pressure wave form in the main pulmonary artery were obtained in all patients, and from the branches of the pulmonary artery in 31 . Recordings were usually made at a paper speed of $8 \mathrm{~cm}$. per sec., using maximum sensitivity and, except in Case 10, there was no electrical damping. Pulmonary capillary pressures were obtained in the usual way by "wedging"; where this failed owing to the size of the Telco instrument, an ordinary single lumen catheter was used. Blood gas analysis was carried out in all cases, and the cardiac output was measured in 33 patients by the Fick principle. 
Fig. 4.-Pressure recordings obtained from the right ventricle of two patients with atrial septal defects. A, from Case 17 , shows a presystolic wave with peak 0.16 second after the beginning of the $P$ wave of the electrocardiogram, and B, from Case 20, with the peak of the presystolic wave $0 \cdot 13$ 0.14 second after the beginning of the $P$ wave. The scale is shown in $B$.

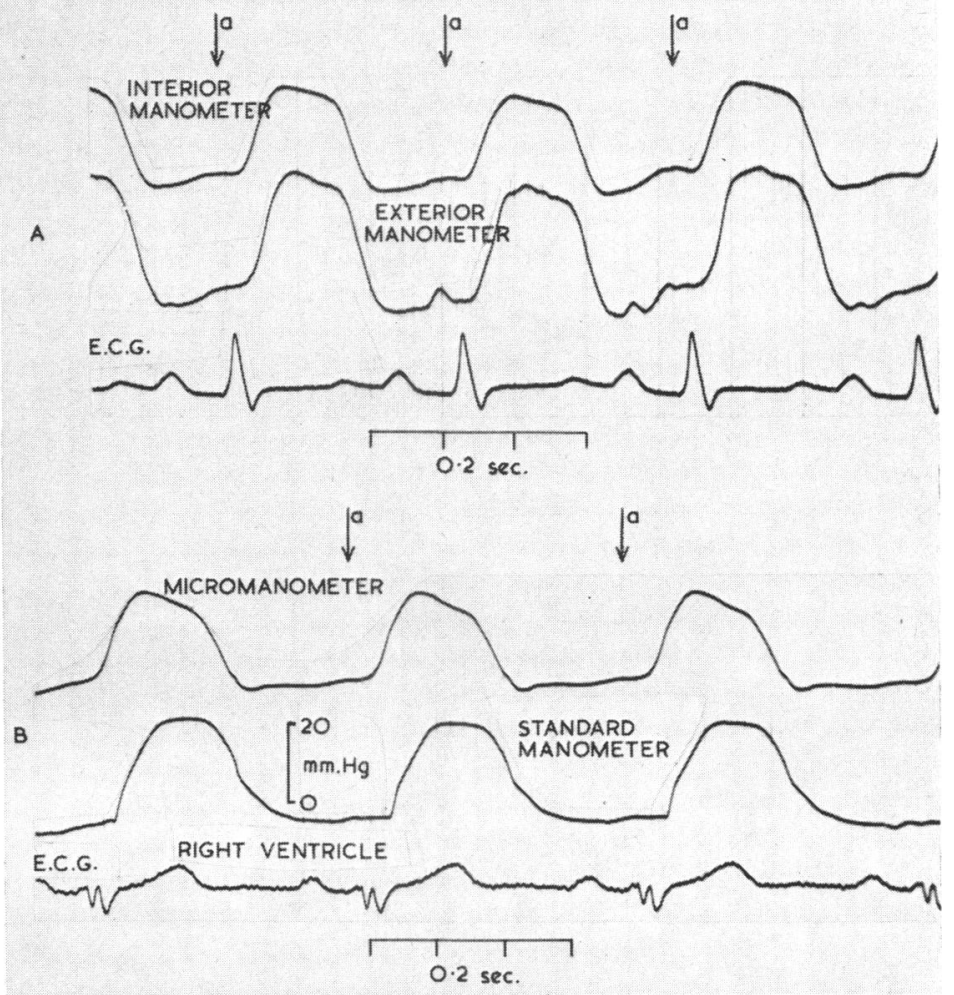

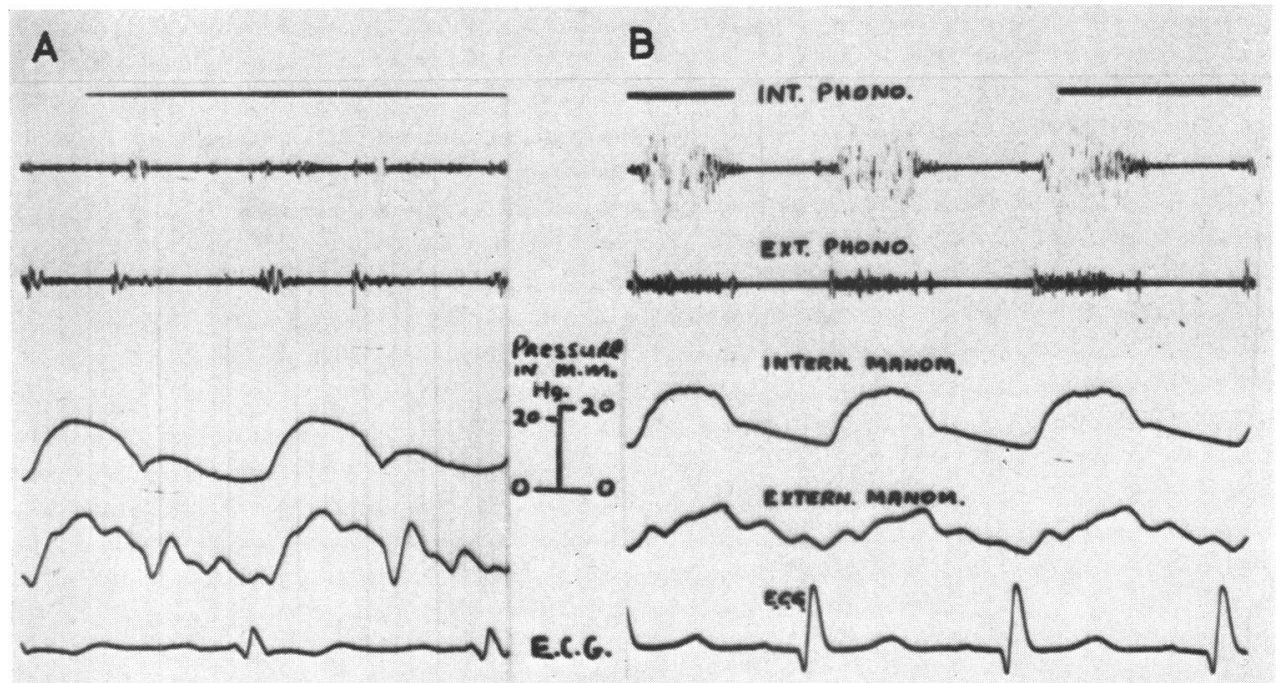

FIG. 5.-A comparison of pressure recordings obtained from the main pulmonary artery of two patients with increased pulmonary blood flow. A, from Case 11 with a normal pulmonary valve shows a dicrotic wave of $2 \mathrm{~mm}$. $\mathrm{Hg}$, whereas B, from Case 16 with pulmonary stenosis has virtually no dicrotic wave. The top tracing in $B$ is the intracardiac phonocardiogram which shows the pulmonary systolic murmur and delayed pulmonary second sound. 


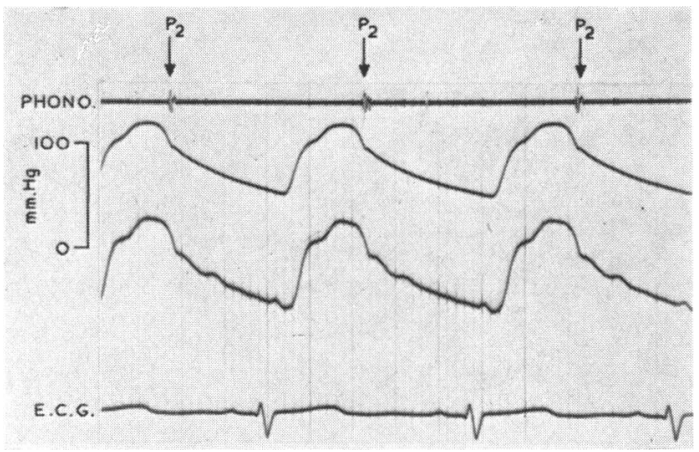

FIG. 6.-Pulmonary arterial pressure recording from a patient with pulmonary hypertension associated with a ventricular septal defect (Case 27; pulmonary artery pressure $=125$ / $60 \mathrm{~mm}$. Hg). There are no dicrotic or secondary waves. The upper pressure tracing is from the internal manometer.

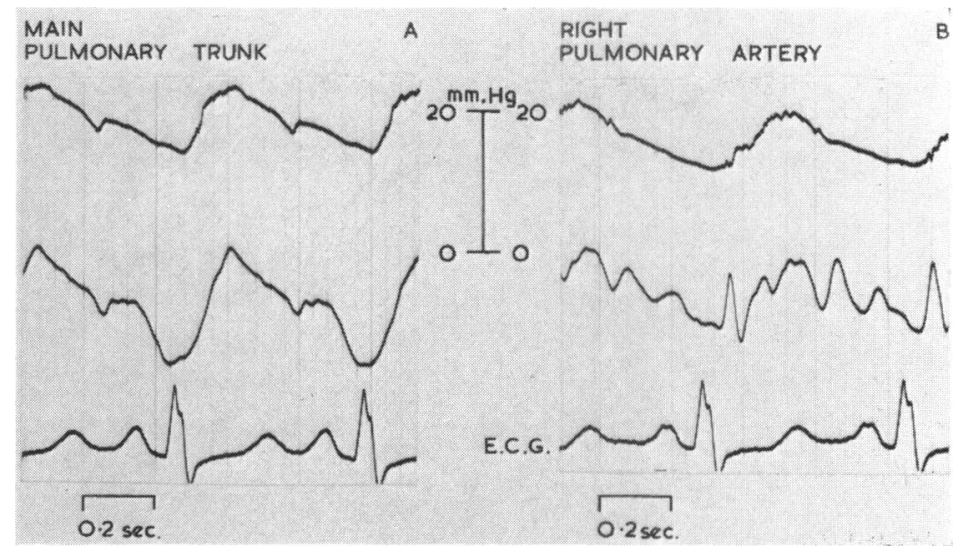

Fig. 7.-There is a clear dicrotic wave in the pressure recording from the main pulmonary trunk (A), but this is virtually absent in recording (B) from the right branch of the pulmonary artery (Case 24). In each case the upper pressure tracing is from the internal manometer and the lower tracing is from the external manometer.

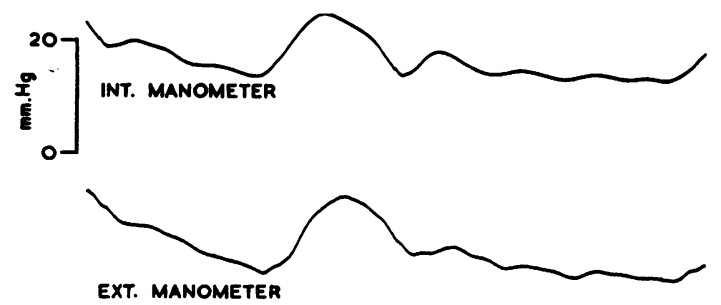

Fig. 8.-Pulmonary artery pressure tracings from Case 7 showing secondary pressure waves following the dicrotic wave. The secondary waves are large and clear and in Tables I and II would be denoted "t".

E.C.G.

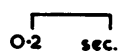




\section{RESULTS}

The Presystolic Wave. The shape of the diastolic part of the pulmonary arterial pressure trace was found to be remarkably constant in individual patients. Usually at least two waves could be seen to be superimposed upon the natural decline in pressure after pulmonary valve closure: an initial undulation just after the pulmonary incisura, and another immediately before the next systole. The first parallels the dicrotic wave of the systemic circulation and is a common finding during routine cardiac catheterization even when the normal fluid-filled catheter system is used. The late pressure wave is less well recognized. It always occurs in immediate presystole (Fig. 3) even if the length of the cardiac cycle alters noticeably between beats. It precedes or is simultaneous with the $R$ deflection of the cardiogram and its peak is approximately $0 \cdot 14-0.18 \mathrm{sec}$. after the start of the $\mathbf{P}$ wave. From its timing it is likely to be produced by atrial systole, following the "a" wave in the right auricle by $0.02-0.04 \mathrm{sec}$. and a presystolic pressure wave in the right ventricle by 0.01-0.02 sec. (Fig. 4).

The Dicrotic Wave. A dicrotic wave was visible in 35 out of the 43 patients studied, in some instances rising as high as $5 \mathrm{~mm}$. $\mathrm{Hg}$ above the incisura pressure (Tables I and II). It was largest in patients with an increased pulmonary blood flow and a normal pulmonary arterial pressure. Virtually all those with an increased blood flow and normal pulmonary valves had a dicrotic wave of at least $2 \mathrm{~mm}$. $\mathrm{Hg}$ in height; it was smaller or absent in some of the patients who had pulmonary stenosis in addition to a septal defect (Fig. 5). The dicrotic wave tended to be small or absent in patients with uncomplicated pulmonary valvular stenosis, although the only one studied with uncomplicated infundibular stenosis had a prominent wave (Table II). The dicrotic wave was absent in more than half of the patients with pulmonary hypertension (Fig. 6): the largest wave was seen in a case of common atrioventricular canal (Case 23) in which there was an exceptionally large pulmonary blood flow (about ten times the systemic flow).

Pressure tracings were obtained from the right or left main branches of the pulmonary artery in 27 patients who had clear dicrotic pressure waves in the main pulmonary trunk. In 23 cases the

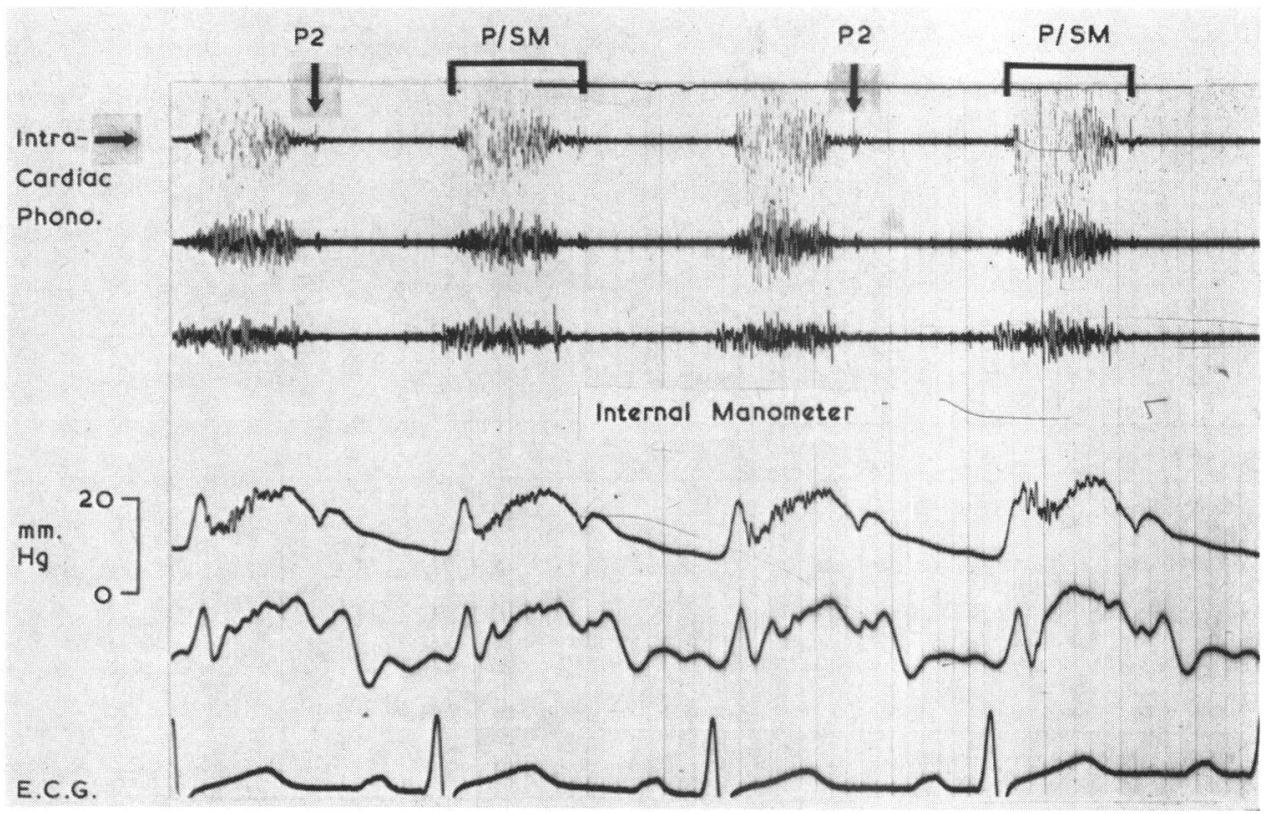

FIG. 9.-Pulmonary arterial pressure tracing from Case 38 showing very small secondary pressure waves during diastole; these would be denoted \pm in Tables I and II. 
amplitude of the wave was smaller in the branches than in the trunk (Fig. 7), in three cases it was the same, and in one case it was larger. In no case was a dicrotic wave seen in one of the branches of the pulmonary artery, when none was present in the main trunk.

Secondary Dicrotic Waves. In some patients with a slow heart rate, secondary and even tertiary pressure waves could be seen to follow the dicrotic wave in the pulmonary artery pressure trace in addition to the presystolic or "a" wave (Fig. 8 and 9). These disappeared if the heart rate increased (Fig. 10), because the whole of diastole was taken up by the dicrotic and presystolic waves. However, their form and frequency were very constant despite changes in heart rate provided diastole was long enough to allow them to appear.

The secondary pressure waves usually occurred in patients who had prominent dicrotic waves, and were not seen in those with small or no dicrotic waves (Fig. 11). They were of similar length to that of the dicrotic wave, and like this wave their amplitude was less in tracings obtained from branches of the pulmonary artery than in those obtained from the pulmonary trunk.

When first seen it was thought that the secondary waves must be artefacts produced by catheter movement. However, this explanation appears unlikely because (1) the wave form was unaffected by slight movement of the position of the catheter in the pulmonary artery; (2) the oscillations were recorded consistently throughout a two-hour catheter session; (3) in the 10 patients in whom secondary waves were recorded in the branches of the pulmonary artery, the wave length was the same as that recorded in the pulmonary trunk, whereas catheter whip usually alters moderately as the

TABLE I

FindiNGS IN GROUP I

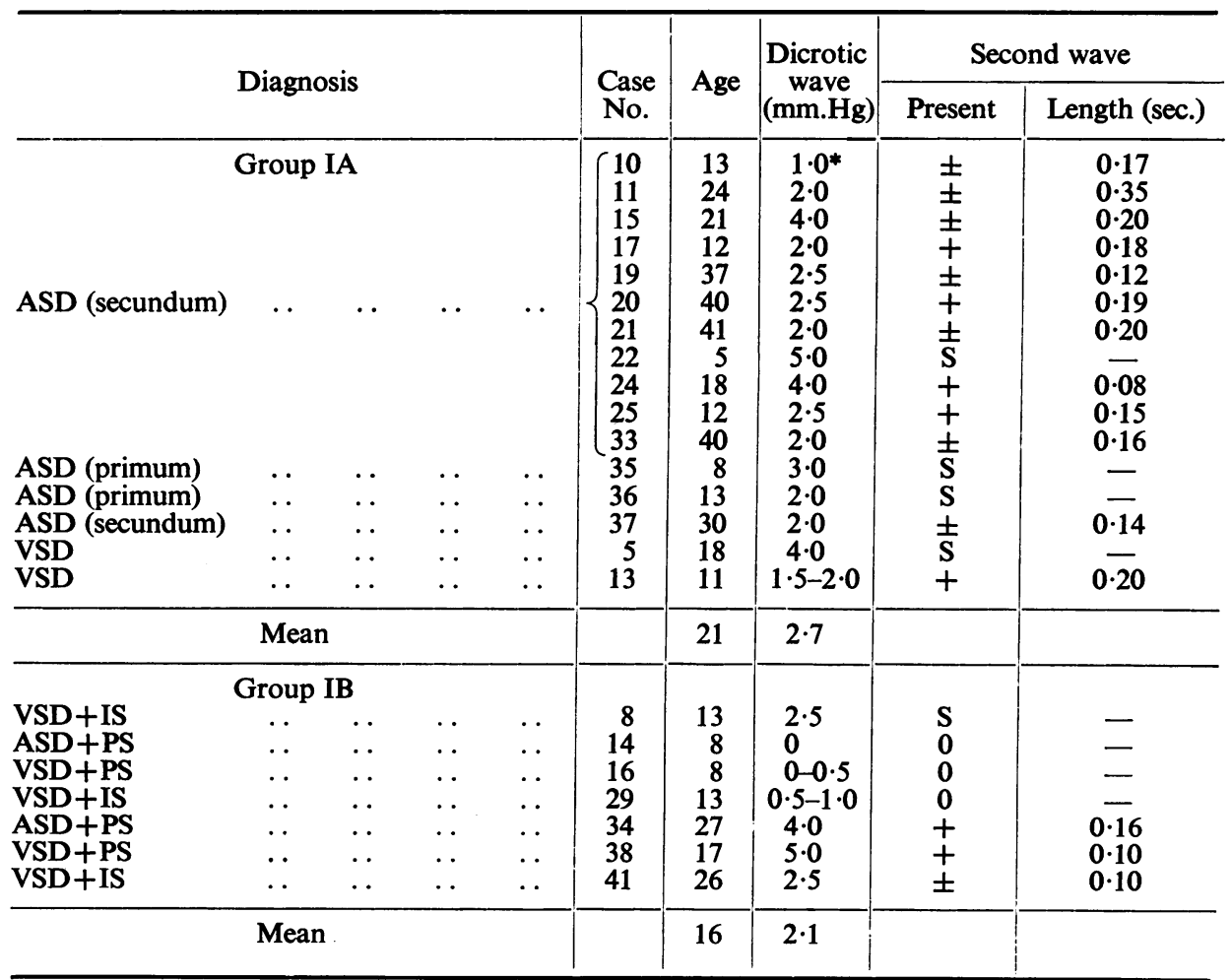

ASD = Atrial septal defect; VSD = Ventricular septal defect; PS = Pulmonary stenosis; IS =Infundibular stenosis *=Electrical damping; $\mathrm{S}=$ Short diastole.

Clear secondary waves denoted by $t$, and very small waves by \pm . 
TABLE II

FindiNGS IN GROUP II

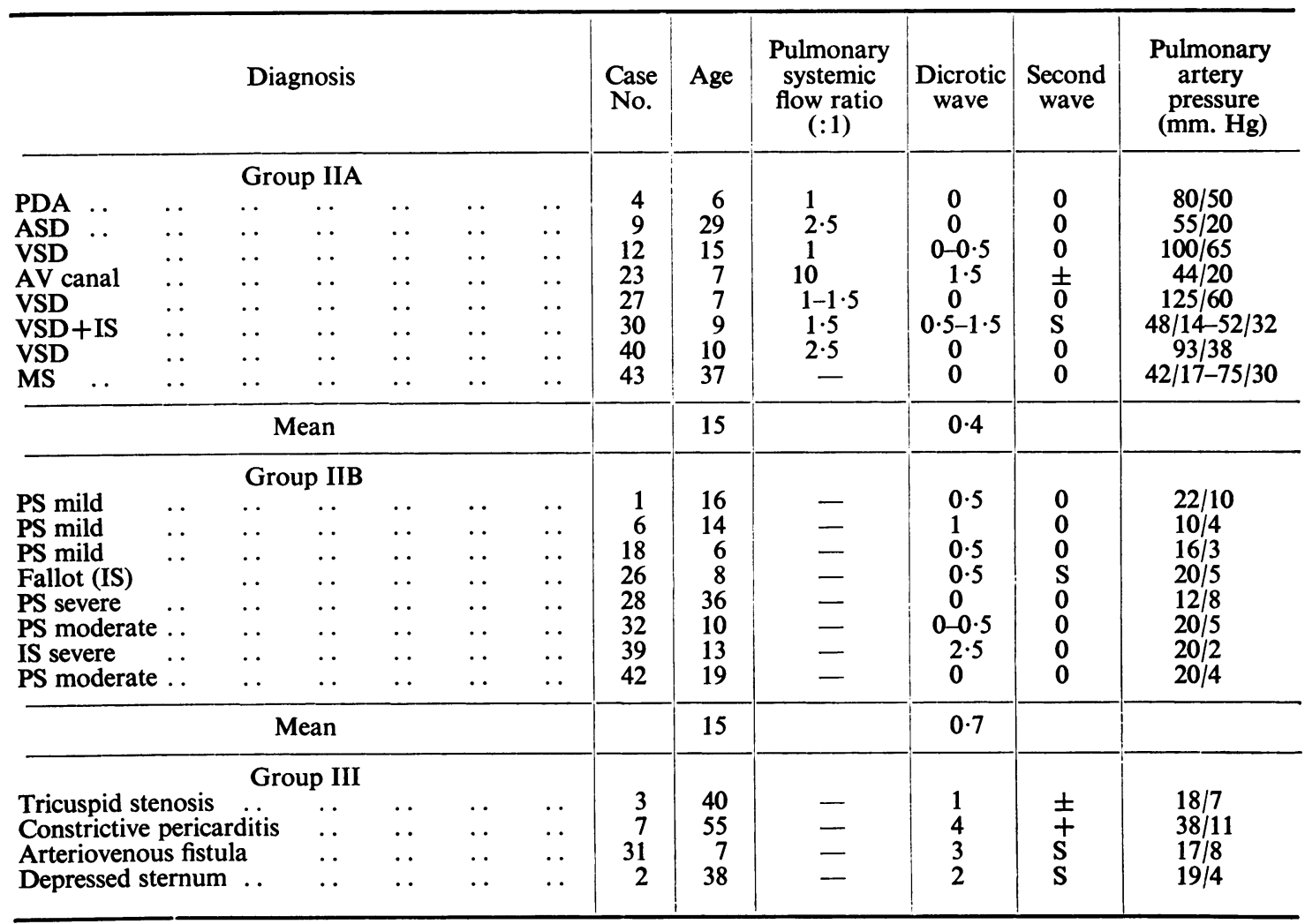

Symbols and abbreviations as for Table $\mathrm{I}$; in addition $\mathrm{PDA}=$ Patent ductus arteriosus; $\mathrm{AV}=$ atrioventricular; MS = Mitral stenosis.

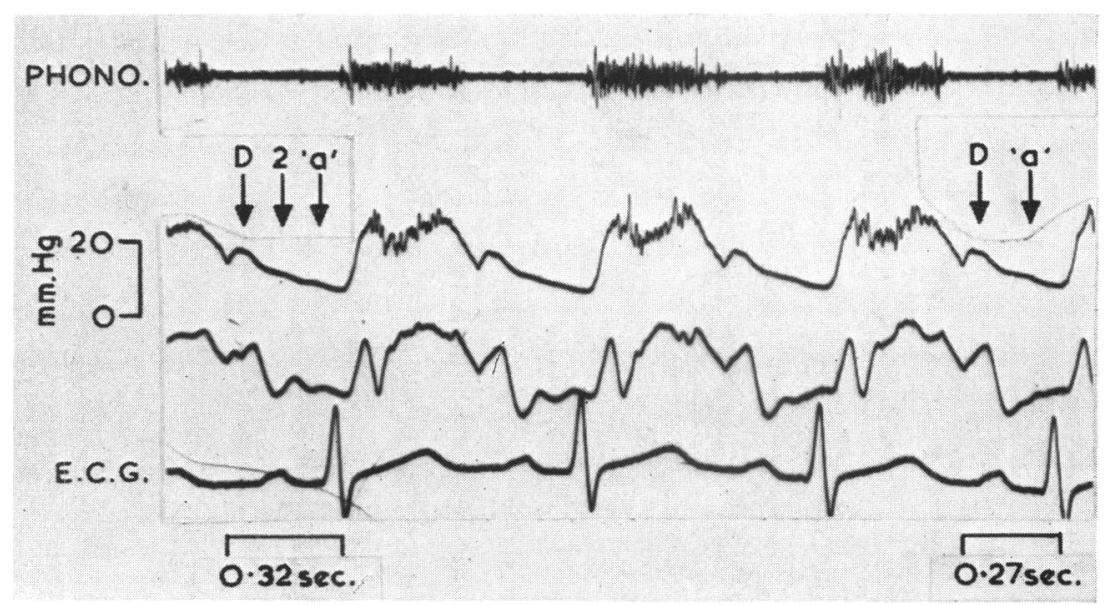

FIG. 10.-Pulmonary arterial pressure tracings from Case 38 showing a small secondary wave (2) between the dicrotic wave (D) and the presystolic wave ('a') in the first cycle, but disappearing in the following cardiac cycles as diastole becomes shorter. 
catheter is pushed into the periphery of the lung; and (4) banging and to and fro movement of the catheter outside the body produced pressure oscillations in the recording obtained with the external manometer system but no such artefacts in the tracing from the catheter tip manometer.

Clear secondary waves were present in 8 of the patients studied (denoted + in Tables I and II), very small secondary waves (Fig. 9) were present in 10 patients (denoted \pm in Table I and II), and no secondary waves were seen in 17 patients. In 9 patients it was not possible to determine whether additional waves were present because diastole was too short. Secondary pressure waves were seen in all patients in Group IA who had slow heart rates. The secondary waves were a variable finding, like the dicrotic waves, in tracings from patients who had pulmonary stenosis in addition to an increased pulmonary blood flow (Group IB). With the exception of the patient with an atrio-ventricular canal and very large pulmonary flow, secondary waves were absent in the patients in Group II with pulmonary hypertension or pulmonary stenosis and a normal pulmonary blood flow.

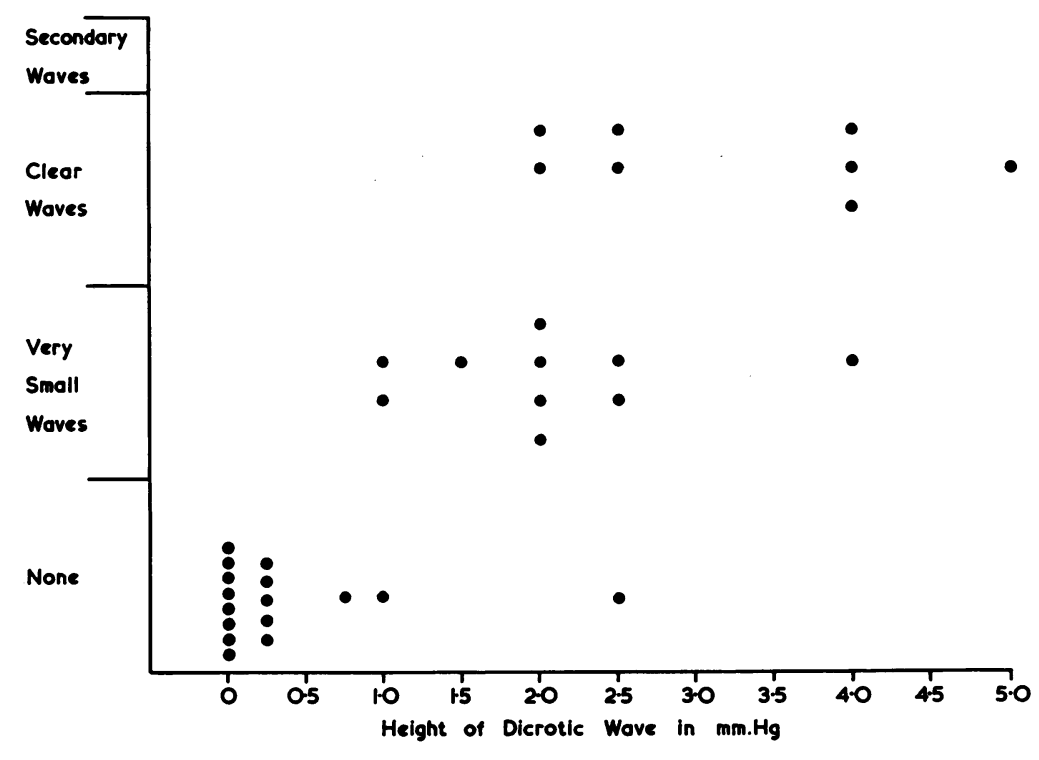

FIG. 11.-The incidence of secondary pressure waves compared to the height of the dicrotic wave in 34 patients.

\section{Discussion}

Wiggers in 1914 reported the presence of a small dicrotic wave occurring immediately after pulmonary valve closure in the pulmonary arterial pressure tracings of dogs. On occasions further pressure waves following the dicrotic wave have been recorded, but in view of the high inertia of the instruments used, these waves have been regarded as superimposed artefacts (Johnson et al., 1937; Wiggers, 1952). For reasons already presented, however, the secondary wave which has been seen to follow the dicrotic wave in some tracings from patients in the present study is unlikely to be an artefact.

The results obtained here suggest that the additional pressure waves are secondary oscillations induced by the dicrotic wave, because they have a similar frequency and rarely occur unless the dicrotic wave is large. It is possible that the dicrotic wave and other pressure oscillations could be initiated by the pulse wave being reflected from the periphery of the pulmonary vascular system. However, on theoretical grounds, Engelberg and Dubois (1959) considered that the wave-length of the pulmonary arterial pressure wave was too long in relation to the length of the vascular system to permit reflected waves. Further, if the oscillations were due to reflected pressure waves, their 
frequency, as deduced from the length of the vessels and the speed of transmission of pressure waves in the pulmonary system (Bramwell and Hill, 1922; Engelberg and Dubois, 1959) might be expected to be far greater than that observed in the present study.

The dicrotic and secondary pressure waves were found to become progressively smaller towards the periphery of the lung, which implies either that they are standing waves or that they are produced in the region of the pulmonary trunk. The dicrotic wave in the aorta has been attributed to movement of the aortic valve cusps, and by analogy pressure oscillations might be produced in the pulmonary artery by movements of the pulmonary valve (Wiggers, 1914; 1921). Certainly the finding in the present study that the dicrotic and secondary waves were small or absent in patients with uncomplicated pulmonary stenosis suggests that the pulmonary valve plays a part in their production. However, there must be other factors involved because prominent dicrotic waves were seen in two patients with pulmonary stenosis in addition to an increased pulmonary blood flow from an intracardiac shunt. Possibly it is the character of right ventricular ejection that determines the size of the dicrotic wave: the slow ejection spread throughout systole, which is associated with pulmonary stenosis, tending to reduce the size of the dicrotic wave, whereas the abnormally rapid ejection associated with an increased pulmonary blood flow would give rise to a large wave.

The absence of dicrotic and secondary pressure waves in most of the patients with pulmonary hypertension implies that these are suppressed by a high pulmonary vascular resistance. However, at least one subject (Case 43) with pulmonary hypertension and no dicrotic or secondary waves had a normal vascular resistance. It seems probable therefore that other factors, such as the elasticity and the inertia of the pulmonary system, are important in determining the presence or absence of pressure waves. The elasticity of the system is likely to be reduced both by the pathological changes in the vessel walls, which may accompany pulmonary hypertension, and the abnormal degree of stretch to which the vessel walls are subjected in this condition. The effect of pathological processes upon inertia in the pulmonary system is less easy to predict. But it is likely to depend mainly upon the flow of blood in the pulmonary vessels, since Patel, Schilder, and Mallos (1960) have shown that, at least in dogs, there is little inertia in the pulmonary artery wall.

The frequency of the pressure waves occurring in the pulmonary artery during diastole appeared to be independent of the heart rate. Further, it did not differ between patients who had a septal defect alone and those who had a septal defect associated with pulmonary stenosis. Presumably, therefore, the frequency is determined by the physical characteristics of the pulmonary vascular system rather than by factors modifying ventricular emptying. Theoretically it might be expected that the less elastic the arteries the higher would be the frequency, and hence that the frequency would increase with age. In the present study no correlation could be shown between the frequency of the diastolic pressure waves, and the age of the patient. However, it may be that too few patients were studied to demonstrate an association.

\section{SUMMARY}

The pulmonary artery wave form during diastole has been studied in 43 patients using a cathetertip micromanometer.

A small presystolic wave was seen in 30 patients, and from its timing it seems likely that it reflected contraction of the right atrium. A clear dicrotic wave was present in 35 of the subjects. It was large in patients with an increased pulmonary blood flow due to an atrial or ventricular septal defect, and small or absent in patients with pulmonary hypertension or pulmonary stenosis.

A small secondary pressure wave was seen to follow the dicrotic wave in 18 patients. These extra waves were only seen in patients who had large dicrotic waves, and both they and the dicrotic waves tended to be smaller in pressure tracings obtained from the branches of the pulmonary artery.

The findings imply that the dicrotic and secondary waves have a common origin in the main pulmonary trunk, and possible mechanisms for their production are discussed. It is suggested 
that in the presence of a normal pulmonary valve, the size of the dicrotic wave and the presence or absence of secondary pressure waves depend upon the hæmodynamic characteristics of the pulmonary vascular system.

I am deeply indebted to Dr. D. W. Barritt for carrying out the majority of the cardiac catheterizations and to him and Dr. D. H. Davies for making this work possible. Mr. L. Clarke is to be thanked for his point device assistance.

\section{REFERENCES}

Bouchard, F., and Cornu, C. (1954). Arch. Mal. Caur, 47, 417.

Bramwell, J. C., and Hill, A. V. (1922). Proc. roy. Soc., Series B, 93, 298.

Ellis, E. J., Gauer, O. H., and Wood, E. H. (1951). Circulation, 3, 390.

Engelberg, J., and Dubois, A. B. (1959). Amer. J. Physiol., 196, 401.

Frank, O. (1895). Z. Biol., 32, 370.

Howell, W. H., and Donaldson, F. (1884). Phil. Trans. London, 175, 139.

Johnson, V., Hamilton, W. F., Katz, L. N., and Weinstein, W. (1937). Amer. J. Physiol., 120, 624.

Norman, J. (1958). Soc. card. Techn. J., 3, 197.

Patel, D. J., Schilder, D. P., and Mallos, A. J. (1960). J. appl. Physiol., 15, 92.

Rashkind, W. J. (1960). Amer. Heart J., 59, 36.

Rodbard, S., and Shaffer, A. B. (1956). Amer. Heart J., 51, 885.

Soulié, C., Laurens, P. Allard, E., and Bouchard, F. (1954). 2nd World Congress of Cardiology, Washington. In Acta cardiol., 9, 689.

Wiggers, C. J. (1914). Amer. J. Physiol., 35, 124.

- (1921). Amer. J. Physiol., 56, 415.

- (1952). Circulatory dynamics: physiologic studies. Modern Medical Monographs, No. 4. Grune and Stratton, New York. 